\title{
Phase Transitions in Disordered Systems: The Example of the Random-Field Ising Model in Four Dimensions
}

\author{
Nikolaos G. Fytas, ${ }^{1}$ Víctor Martín-Mayor, ${ }^{2,3}$ Marco Picco, ${ }^{4}$ and Nicolas Sourlas ${ }^{5}$ \\ ${ }^{1}$ Applied Mathematics Research Centre, Coventry University, Coventry CV1 5FB, United Kingdom \\ ${ }^{2}$ Departamento de Física Téorica I, Universidad Complutense, 28040 Madrid, Spain \\ ${ }^{3}$ Instituto de Biocomputacíon y Física de Sistemas Complejos (BIFI), 50009 Zaragoza, Spain \\ ${ }^{4}$ LPTHE (Unité mixte de recherche du CNRS UMR 7589), Université Pierre et Marie Curie-Paris 6, \\ 4 place Jussieu, 75252 Paris Cedex 05, France \\ ${ }^{5}$ Laboratoire de Physique Théorique de l'Ecole Normale Supérieure (Unité Mixte de Recherche du CNRS et de l'Ecole Normale \\ Supérieure, associée à l'Université Pierre et Marie Curie, PARIS VI) 24 rue Lhomond, 75231 Paris Cedex 05, France
}

(Received 23 February 2016; published 3 June 2016)

\begin{abstract}
By performing a high-statistics simulation of the $D=4$ random-field Ising model at zero temperature for different shapes of the random-field distribution, we show that the model is ruled by a single universality class. We compute to a high accuracy the complete set of critical exponents for this class, including the correction-to-scaling exponent. Our results indicate that in four dimensions (i) dimensional reduction as predicted by the perturbative renormalization group does not hold and (ii) three independent critical exponents are needed to describe the transition.
\end{abstract}

DOI: 10.1103/PhysRevLett.116.227201

Introduction.-The random-field Ising model (RFIM) [1] is maybe the simplest disordered system in physics [2]. Applications in hard and soft condensed matter physics are many (see, e.g., Refs. [3-5]), and their numbers increase [6-8]. The RFIM Hamiltonian is

$$
\mathcal{H}=-J \sum_{\langle x y\rangle} S_{x} S_{y}-\sum_{x} h_{x} S_{x}
$$

with the spins $S_{x}= \pm 1$ occupying the nodes of a hypercubic lattice in space dimension $D$ with nearestneighbor ferromagnetic interactions and $h_{x}$ independent random magnetic fields with zero mean and dispersion $\sigma$.

The renormalization group (RG) suggests that $D$ is an all-important variable (no less than temperature $T$ ) [9]. Indeed, at low temperature $T$ and for small-enough disorder (i.e., $\sigma \ll J$ ), we encounter the ferromagnetic phase, provided that $D \geq 3[10,11]$. A phase transition to a disordered, paramagnetic phase occurs upon increasing $T$ or $\sigma$. Yet, for $D=2$, the tiniest $\sigma>0$ suffices to destroy the ferromagnetic phase [12]. Furthermore, perturbative RG (PRG) computations, employing the mathematically unorthodox replica trick to restore the translation invariance broken by disorder [13], tell us that the upper critical dimension is $D_{u}=6$ [14] (the mean field is quantitatively accurate if $D>D_{u}$ ).

The RFIM and branched polymers are unique among disordered systems: a supersymmetry [15] makes it possible to analyze the PRG to all orders of perturbation theory [16]. Supersymmetry predicts dimensional reduction: the RFIM critical behavior in dimension $D$ would be the same as of a nondisordered ferromagnet in dimension $D-2[15,17]$. Yet, see above, the RFIM orders in $D=3$ while the ferromagnet in $D=1$ does not.

The failure of the PRG begs the question: Is there an intermediate dimension $D_{\text {int }}<D_{u}$ such that the PRG is accurate for $D>D_{\text {int }}$ ? The issue is obviously relevant to all disordered systems [18].

Yet, the RFIM is a peculiar disordered system. The relevant RG fixed point is believed to lie at $T=0$ [22-24]. Therefore, in order to describe the critical behavior one needs three independent critical exponents and two correlation functions, namely, the connected and disconnected propagators, $C_{x y}^{(\mathrm{con})}$ and $C_{x y}^{(\mathrm{dis})}$ [25]. At the critical point and for large $r$ ( $r$ : distance between $x$ and $y$ ), they decay as

$C_{x y}^{(\text {con })} \equiv \frac{\partial \overline{\left\langle S_{x}\right\rangle}}{\partial h_{y}} \sim \frac{1}{r^{D-2+\eta}} ; \quad C_{x y}^{(\mathrm{dis})} \equiv \overline{\left\langle S_{x}\right\rangle\left\langle S_{y}\right\rangle} \sim \frac{1}{r^{D-4+\bar{\eta}}}$,

where the $\langle\cdots\rangle$ are thermal mean values as computed for a given realization, a sample, of the random fields $\left\{h_{x}\right\}$. The overline refers to the average over the samples. The relationship between the anomalous dimensions $\eta$ and $\bar{\eta}$ is hotly debated, and it is one of our main themes here, as it entails the correct parametrization of the neutron-scattering line shape [26,27]. Supersymmetry predicts $\eta=\bar{\eta}$.

We also recall phenomenological scaling as an alternative to the PRG $[1,22-24]$. The prediction $\bar{\eta}=2 \eta$ by Schwartz and co-workers [28-30], although not a consequence of phenomenological scaling, has gained ground throughout the years. However, Tarjus and co-workers [31-33] have suggested that rare events, neglected in Refs. [28-30], spontaneously break supersymmetry at 
the intermediate dimension $D_{\text {int }} \approx 5.1$. For $D>D_{\text {int }}$ replica predictions hold: supersymmetry is valid and $\bar{\eta}=\eta$. For $D<D_{\text {int }}$, instead, there are three independent critical exponents.

Unfortunately, both the perturbative and the phenomenological RG approaches lack predictions allowing for detailed comparisons with experiments. In this context numerical simulations become a crucial tool. This is especially true at $T=0$, where fast polynomial algorithms $[34,35]$ allow us to find exact ground states for a wide range of accessible system sizes $L$. This approach has been used mainly at $D=3[36-48]$ but also for higher dimensions on a smaller scale [38,49-51], although having a strong command over the $D$ dependency of the random-field criticality would be desirable and is the motivation of the current work.

Noteworthy, claims of universality violations for the RFIM at $D \geq 3$ have been quite frequent when comparing different distributions of random fields [37-40]. Fortunately, using new techniques of statistical analysis [5], it has been possible to show that, at least in $D=3$, these apparent universality violations are merely finite-size corrections to the leading scaling behavior $[47,52]$. We also note the numerical bound $2 \eta-\bar{\eta} \leq 0.0026$ (10) [47] which is valid in $D=3$ [53].

Here, we report the results of large-scale zero-temperature numerical simulations at $D=4$. Our state-of-the-art analysis $[5,47]$ provides high-accuracy estimates for the critical exponents $\eta, \bar{\eta}$, and $\nu$, as well as for other RG invariants, indicating that dimensional reduction does not hold at this particular dimensionality. A clear case for universality is made by comparing Gaussian- and Poissonian-distributed random fields, but only after taming the strong scaling corrections. Finally, we present overwhelming numerical evidence in favor of $2 \eta-\bar{\eta}>0$, indicating that three independent critical exponents are needed to describe the transition and, furthermore, that the intermediate space dimension where supersymmetry gets restored is larger than four.

Simulation details and finite-size scaling.-We consider the Hamiltonian Eq. (1) on a $D=4$ hypercubic lattice with periodic boundary conditions and energy units $J=1$. Our random fields $h_{x}$ follow either a Gaussian $\left(\mathcal{P}_{G}\right)$, or a Poissonian $\left(\mathcal{P}_{P}\right)$ distribution:

$$
\begin{aligned}
& \mathcal{P}_{G}(h, \sigma)=\frac{1}{\sqrt{2 \pi \sigma^{2}}} e^{-\left(h^{2} / 2 \sigma^{2}\right)}, \\
& \mathcal{P}_{P}(h, \sigma)=\frac{1}{2|\sigma|} e^{-(|h| / \sigma)},
\end{aligned}
$$

where $-\infty<h<\infty$. For both distributions $\sigma$ is our single control parameter.

We simulated lattice sizes from $L=4$ to $L=60$. For each $L$ and $\sigma$ value we computed ground states for $10^{7}$ samples, see the Supplemental Material [54]. For comparison, 3200 samples of $L=32$ were simulated in Ref. [49] and 5000 samples of $L=64$ in Ref. [50].

From simulations at a given $\sigma$, we computed $\sigma$ derivatives and extrapolated to neighboring $\sigma$ values by means of a reweighting method [5]. We computed the secondmoment correlation length [55] for each of the two propagators $C^{(\mathrm{con})}$ and $C^{(\mathrm{dis})}$ in Eq. (2), $\xi^{(\mathrm{con})}$ and $\xi^{(\mathrm{dis})}$, as well as the corresponding susceptibilities $\chi^{(\mathrm{con})}$ and $\chi^{(\mathrm{dis})}$. We also computed the dimensionless Binder ratio $U_{4}=$ $\overline{\left\langle m^{4}\right\rangle} / \overline{\left\langle m^{2}\right\rangle^{2}}$ and the ratio $U_{22}=\chi^{(\mathrm{dis})} /\left[\chi^{(\mathrm{con})}\right]^{2}$ that gives direct access to the difference of the anomalous dimensions $2 \eta-\bar{\eta}$. For additional technical details see Ref. [5].

We followed the quotients-method approach to finitesize scaling [55-57]. In this method, one considers dimensionless quantities $g(\sigma, L)$ that, barring correction to scaling, are $L$ independent at the critical point. We consider two such $g$, namely $\xi^{\text {(dis) }} / L$ and $\xi^{(\text {con })} / L$ (also $U_{4}$
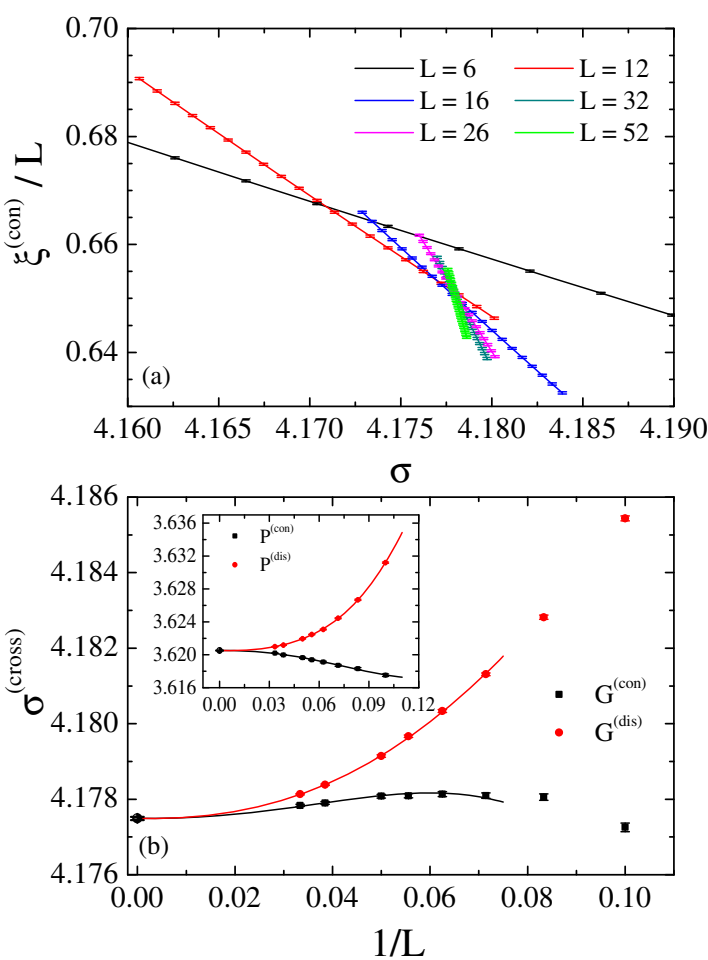

FIG. 1. Top (a): Connected correlation length in units of the system size $L$ vs $\sigma$ (we show data only for some characteristic $L$ values for clarity). Because of scale invariance, all curves should cross at the critical point $\sigma_{\mathrm{c}}$. Yet, small systems deviate from the large- $L$ scale-invariant behavior. Bottom (b): For Gaussian random fields, crossing points $\sigma_{\mathrm{c}, L}$ of the pair of lattice sizes $(L, 2 L)$ for $\xi^{(\mathrm{dis})} / L$ and $\xi^{(\mathrm{con})} / L$, as a function of $1 / L$. Lines are fits to Eq. (6), constrained to yield a common extrapolation to $L=\infty$ (depicted as a black circle at the origin in this figure and in the following ones). Inset: Same as in bottom panel, but for the case now of Poissonian random fields. In all figures the notation $\mathrm{G}^{\text {(con),(dis) }}$ [or $\mathrm{P}^{\text {(con),(dis) }}$ ] distinguishes the type of crossing point (or the type of random fields, i.e., Gaussian or Poissonian). 
is dimensionless). Given a dimensionless quantity $g$, we consider a pair of lattices sizes $L$ and $2 L$ and determine the crossing $\sigma_{\mathrm{c}, L}$, where $g\left(\sigma_{\mathrm{c}, L}, L\right)=g\left(\sigma_{\mathrm{c}, L}, 2 L\right)$, see Fig. 1(a). For each random-field distribution we compute two such $\sigma_{\mathrm{c}, L}$, one for $\xi^{\text {(dis) }} / L$ and one for $\xi^{(\mathrm{con})} / L$. Crossings approach the critical point as $\sigma_{\mathrm{c}}-\sigma_{\mathrm{c}, L}=\mathcal{O}\left(L^{-(\omega+1 / \nu)}\right)$, with $\omega$ being the leading corrections-to-scaling exponent.

Dimensionful quantities $O$ scale with $\xi$ in the thermodynamic limit as $\xi^{x_{O} / \nu}$, where $x_{O}$ is the scaling dimension of $O$. At finite $L$, we consider the quotient $Q_{O, L}=O_{2 L} / O_{L}$ at the crossing (for dimensionless magnitudes $g$, we write $g_{L}^{\text {cross }}$ for either $g_{L}$ or $g_{2 L}$, whichever shows less finite-size corrections)

$$
Q_{O, L}^{\text {cross }}=2^{x_{O} / \nu}+O\left(L^{-\omega}\right) ; \quad g_{L}^{\text {cross }}=g^{*}+O\left(L^{-\omega}\right) .
$$

$Q_{O}^{\text {cross }}$ (or $g_{L}^{\text {cross }}$ ) can be evaluated both at the crossing for $\xi^{\text {(dis) }} / L$ or $\xi^{(\text {con })} / L$. The two choices differ only in the scaling corrections, an opportunity we shall use. The RG tells us that $x_{O}, g^{*}, \omega$, and $\nu$, are universal. We shall compute the critical exponents using Eq. (4) with the following dimensionful quantities: $\sigma$ derivatives $\left[x_{D_{\sigma} \xi^{\text {(con })}}=\right.$ $\left.x_{D_{\sigma} \xi^{\text {(dis })}}=1+\nu\right]$, susceptibilities $\left[x_{\chi^{(\mathrm{con})}}=\nu(2-\eta)\right.$ and $\left.x_{\chi^{(\mathrm{dis})}}=\nu(4-\bar{\eta})\right]$, and the ratio $U_{22}\left[x_{U_{22}}=\nu(2 \eta-\bar{\eta})\right]$. We also note the ambiguity with $g_{L}^{\text {cross }}$. If you study, say, $g=\xi^{\text {(dis) }} / L$ at the crossings of $\xi^{(\text {con })} / L$, you may focus just as well on $g_{2 L}$, or on $g_{L}$. Scaling corrections can be the smallest in either case. The corrections-minimizing choices are $g_{L}^{\text {cross }}=g_{2 L}$ for $\xi^{(\text {dis })} / L, g_{L}^{\text {cross }}=g_{L}$ for $\xi^{(\text {con })} / L$, and $g_{L}^{\text {cross }}=g_{2 L}$ for $U_{4}$.

Now, an important issue is evinced in Fig. 1(b): The size evolution is nonmonotonic (for a spectacular example see Fig. 1, Supplemental Material [54]). In other words, our accuracy is enough to resolve subleading corrections to scaling.
We take into account subleading corrections in an effective way. Let $X_{L}$ be either $g_{L}^{\text {cross }}$ or the effective scaling dimension $x_{O}^{\text {(eff) }} / \nu=\log Q_{O}^{\text {cross }}(L) / \log 2$, recall Eq. (4). We consider two different fits $\left(a_{k}, b_{k}, c_{k}, d_{k}\right.$ for $k=1,2$ are scaling amplitudes). (i) The quadratic fit (QF) which is

$$
\begin{aligned}
X_{L} & =X^{*}+a_{1} L^{-\omega}+a_{2} L^{-2 \omega}, \\
\sigma_{\mathrm{c}, L} & =\sigma_{c}+b_{1} L^{-[\omega+(1 / \nu)]}+b_{2} L^{-[2 \omega+(1 / \nu)]} .
\end{aligned}
$$

(ii) However, $\omega$ turns out to be so large, that $L^{-2 \omega}$ terms (certainly present) are maybe not the most relevant correction. Hence, we consider also the leading + analytic corrections fit $[(L+A) F]$,

$$
\begin{aligned}
X_{L} & =X^{*}+c_{1} L^{-\omega}+c_{2} L^{-(2-\eta)} \\
\sigma_{\mathrm{c}, L} & =\sigma_{c}+d_{1} L^{-[\omega+(1 / \nu)]}+d_{2} L^{-[2-\eta+(1 / \nu)]} .
\end{aligned}
$$

The $L^{-(2-\eta)}$ term is due to the nondivergent analytic background. We plug $2-\eta \simeq 1.8$ in the $(L+A) F$.

Since both fits are well motivated only when $L$ is large enough, we restrict ourselves to data with $L \geq L_{\min }$. To determine an acceptable $L_{\min }$ we employ the standard $\chi^{2}$ test for goodness of fit, where $\chi^{2}$ is computed using the complete covariance matrix. In practice, we found that both types of fit give compatible results. In the following, we present the results of the QF [for the results of the $(L+A) F$, see Table I].

Results.-The procedure we follow is standard by now [58]. The first step is the estimation of the corrections-toscaling exponent $\omega$. Take, for instance, $\xi^{(\mathrm{con})} / L$. For each pair of sizes $(L, 2 L)$ we have four estimators, Fig. 2, top: two crossing points, either $\xi^{(\mathrm{con})} / L$ or $\xi^{\text {(dis) }} / L$, and two disorder distributions. Rather than four independent fits to Eq. (5), we perform a single joint fit: we minimize the combined $\chi^{2}$ goodness of fit, by imposing that the extrapolation to $L=\infty,\left(\xi^{(\mathrm{con})} / L\right)^{*}$, as well as exponent $\omega$ are

TABLE I. Summary of results. The second column is the outcome of a fit to Eq. (5) while the fourth column is obtained fitting to Eq. (7) [yet, critical points $\sigma_{\mathrm{c}}$ were obtained from Eqs. (6) or (8), correspondingly]. The first row reports a joint fit for $\omega, \xi^{(\operatorname{con})} / L$ and $\eta$. The remaining quantities were individually extrapolated to $L=\infty \cdot \chi^{2}$ is the standard figure of merit (DOF: number of degrees of freedom in the fit).

\begin{tabular}{lcccc}
\hline \hline & QF & $\chi^{2} / \mathrm{DOF}$ & $(\mathrm{L}+\mathrm{A}) \mathrm{F}$ & $\chi^{2} / \mathrm{DOF}$ \\
\hline$\omega$ & $1.30(9)$ & & $1.60(14)$ & \\
$\xi^{(\text {con })} / L$ & $0.6584(8)$ & $27.85 / 29$ & $0.6579(+6 /-4)$ & $40.33 / 37$ \\
$\eta$ & $0.1930(13)$ & & $0.1922(10)$ & \\
$\sigma_{\mathrm{c}}(G)$ & $4.17749(4)(2)$ & $5.6 / 7$ & $4.17750(4)(2)$ & $3.2 / 7$ \\
$\sigma_{\mathrm{c}}(P)$ & $3.62052(3)(8)$ & $8.85 / 11$ & $3.62060(3)(1)$ & $9.8 / 11$ \\
$U_{4}$ & $1.04471(32)(14)$ & $10 / 11$ & $1.04490(36)(9)$ & $8.57 / 11$ \\
$\xi^{(\text {dis })} / L$ & $2.4276(36)(34)$ & $16 / 15$ & $2.4225(41)(20)$ & $14 / 15$ \\
$\nu$ & $0.8718(58)(19)$ & $62.9 / 55$ & $0.8688(64)(11)$ & $59.8 / 55$ \\
$2 \eta-\bar{\eta}$ & $0.0322(23)(1)$ & $16.0 / 19$ & $0.0322(25)(1)$ & $16.1 / 19$ \\
\hline \hline
\end{tabular}




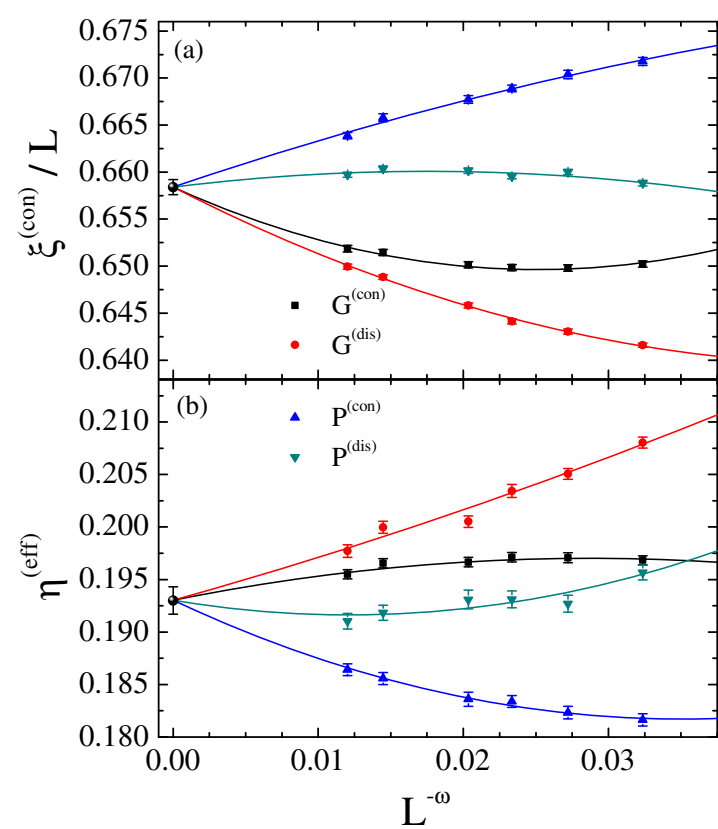

FIG. 2. Top (a): $\xi^{(\operatorname{con})} / L$ vs $L^{-\omega}$ at the crossing points shown in the upper panel of Fig. 1. Bottom (b): The same as in top panel, but for $\eta^{\text {(eff) }}$. Lines correspond to the joint fit reported in Table I.

common for all four estimators (only the scaling amplitudes differ). We judge from the final $\chi^{2}$ value whether or not the fit is fair.

Furthermore, one can perform joint fits for several magnitudes, say $\xi^{(\operatorname{con})} / L$ and $\eta$. Of course, the extrapolation to $L=\infty$ is different for each magnitude, but a common $\omega$ is imposed. However, when we increase the number of magnitudes, the covariance matrix becomes close to singular due to data correlation, and the fit becomes unstable. Therefore, we limit ourselves to $\xi^{(\mathrm{con})} / L$ and $\eta$, see Fig. 2 and Supplemental Material, Fig. 2 [54]. We obtain a fair fit, Table I, by considering pairs $(L, 2 L)$ with $L \geq L_{\min }=14$.

The rest of the quantities of interest are individually extrapolated, following the same procedure, but now fixing $\omega=1.30(9)$. (For the extrapolation of $\xi^{(\mathrm{dis})} / L$ and $U_{4}$ see Supplemental Material Figs. 3 and 4 in Ref. [54].) In fact, the extrapolations in Table I have two error bars. The first error, obtained from the corresponding joint fit to Eq. (5), is of statistical origin. The second error is systematic and takes into account how much the extrapolation to $L=\infty$ changes in the range $1.21<\omega<1.39$.

Our main result is illustrated in Fig. 3, where we show $\log U_{22} / \log 2$ which is a direct measurement of the difference $2 \eta-\bar{\eta}$. This extrapolation is particularly easy, because $L_{\min }=12$ is enough to obtain a good fit and a value $2 \eta-\bar{\eta}=0.0322(24)$. Furthermore, the dependency on $\omega$ of the large- $L$ extrapolation is weak, as shown in Fig. 3, inset. We conclude with high confidence that $2 \eta-\bar{\eta}$ is different than zero, in support of the three-exponent scaling scenario.

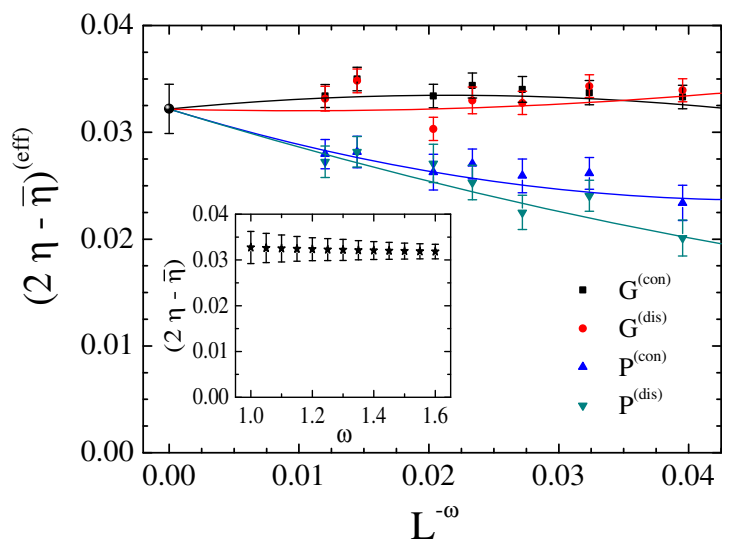

FIG. 3. Effective anomalous dimension difference $2 \eta-\bar{\eta}$ vs $L^{-\omega}$ at the crossing points shown in the upper panel of Fig. 1. Lines correspond to a joint fit to Eq. (5) with $\omega=1.3$. Inset: The extrapolation to large $L$ of $2 \eta-\bar{\eta}$ is essentially $\omega$ independent.

We also determined the effective exponent $\nu^{\text {(eff) }}$ from the $\sigma$ derivatives of $\xi^{(\text {con })}$ and $\xi^{(\text {dis })}$ (see Fig. 5, Supplemental Material [54]). The fits were acceptable even with $L_{\min }=8$ (Table I).

Previous less accurate numerical estimates for the Gaussian distribution of random fields that did not take into account subleading corrections are given by Hartmann: $\nu=0.78(10), \sigma_{\mathrm{c}}=4.18(1), \eta=0.18(1)$, and $\bar{\eta}=0.37(5)$ (so that $2 \eta-\bar{\eta} \approx-0.01$ ) [49] and Middleton: $\nu=0.82(6)$ and $\sigma_{\mathrm{c}}=4.179(2)$ [50]. We may also quote the functional RG estimates $2 \eta-\bar{\eta}=0.08(4), \quad \nu=0.81(3)$ and $\eta=$ 0.24(1) [32], close but incompatible with our findings, probably due to the truncation of the functional $\mathrm{RG}$ equations.

Conclusions.-We have carried out a zero-temperature numerical study of the random-field Ising model in four dimensions. By using two types of the random-field distribution and a proper finite-size scaling scheme we have been able to show universality and to determine with high accuracy the three independent critical exponents, $\eta, \bar{\eta}$, and $\nu$, that are needed to describe the transition, as well as other renormalization group invariants. We stress the nontrivial difference between the anomalous dimensions $2 \eta-\bar{\eta}=0.0322(24)$ which is 10 times larger than its corresponding value at $D=3$ [47]. We thus provided decisive evidence in favor of the three-exponent scaling scenario and the spontaneous supersymmetry breaking [31,32] at some $D_{\text {int }}>4$, against the (restricted) scaling picture [28-30].

Let us conclude by mentioning our preliminary simulations in five dimensions, not reported here. Critical exponents in $D=5$ turn out to be very close to those of the $D=3$ pure Ising ferromagnet, as supersymmetry and dimensional reduction predict. This finding suggests that $D_{\text {int }} \approx 5$, in quantitative agreement with Refs. [31,32]. We intend to pursue this investigation in the near future. As for the suspected upper critical dimension, $D_{u}=6$, 
characteristic logarithmic scaling violations have been reported [51], but still await detailed confirmation. These two final steps will give us access to the full picture of the RFIM scaling behavior.

Our $L=52,60$ lattices were simulated in the MareNostrum and Picasso supercomputers (we thankfully acknowledge the computer resources and assistance provided by the staff at the Red Española de Supercomputación). N.G.F. was supported by Royal Society Research Grant No. RG140201 and from a Research Collaboration Fellowship Scheme of Coventry University. V. M.-M. was supported by MINECO (Spain) through research Contract No. FIS2012-35719C02-01.

[1] Y. Imry and S.-k. Ma, Phys. Rev. Lett. 35, 1399 (1975).

[2] G. Parisi, Field Theory, Disorder and Simulations (World Scientific, Singapore, 1994).

[3] T. Nattermann, in Spin Glasses and Random Fields, edited by A. P. Young (World Scientific, Singapore, 1998).

[4] D. P. Belanger, in Spin Glasses and Random Fields, edited by A. P. Young (World Scientific, Singapore, 1998).

[5] N. G. Fytas and V. Martín-Mayor, arXiv:1512.06571.

[6] S. Franz, G. Parisi, and F. Ricci-Tersenghi, J. Stat. Mech. (2013) L02001.

[7] S. Franz and G. Parisi, J. Stat. Mech. (2013) P11012.

[8] G. Biroli, C. Cammarota, G. Tarjus, and M. Tarzia, Phys. Rev. Lett. 112, 175701 (2014).

[9] K. G. Wilson and J. Kogut, Phys. Rep. 12, 75 (1974).

[10] J. Z. Imbrie, Phys. Rev. Lett. 53, 1747 (1984).

[11] J. Bricmont and A. Kupiainen, Phys. Rev. Lett. 59, 1829 (1987).

[12] M. Aizenman and J. Wehr, Commun. Math. Phys. 130, 489 (1990).

[13] S. F. Edwards and P. W. Anderson, J. Phys. F 5, 965 (1975).

[14] A. Aharony, Phys. Rev. B 18, 3318 (1978).

[15] G. Parisi and N. Sourlas, Phys. Rev. Lett. 43, 744 (1979).

[16] G. Parisi and N. Sourlas, Phys. Rev. Lett. 46, 871 (1981).

[17] A. P. Young, J. Phys. C 10, L257 (1977).

[18] See Refs. [2,19-21] for possible sources of nonperturbative behavior.

[19] M. Mézard and A. P. Young, Europhys. Lett. 18, 653 (1992).

[20] M. Mézard and R. Monasson, Phys. Rev. B 50, 7199 (1994).

[21] G. Parisi and N. Sourlas, Phys. Rev. Lett. 89, 257204 (2002).

[22] J. Villain, J. Phys. (Paris) 46, 1843 (1985).

[23] A. J. Bray and M. A. Moore, J. Phys. C 18, L927 (1985).

[24] D. S. Fisher, Phys. Rev. Lett. 56, 416 (1986).

[25] For $T>0, C_{x y}^{\text {(con) }}=\overline{\left\langle S_{x} S_{y}\right\rangle-\left\langle S_{x}\right\rangle\left\langle S_{y}\right\rangle} / T$, hence the name connected propagator.

[26] Z. Slanič, D. P. Belanger, and J. A. Fernandez-Baca, Phys. Rev. Lett. 82, 426 (1999).

[27] F. Ye, M. Matsuda, S. Katano, H. Yoshizawa, D. Belanger, E. Seppälä, J. Fernandez-Baca, and M. Alava, J. Magn. Magn. Mater. 272-276, Part 2, 1298 (2004); Proceedings of the International Conference on Magnetism (ICM, Rome, 2003).
[28] M. Schwartz and A. Soffer, Phys. Rev. B 33, 2059 (1986).

[29] M. Schwartz, M. Gofman, and T. Natterman, Physica (Amsterdam) 178A, 6 (1991).

[30] M. Gofman, J. Adler, A. Aharony, A. B. Harris, and M. Schwartz, Phys. Rev. Lett. 71, 1569 (1993).

[31] M. Tissier and G. Tarjus, Phys. Rev. Lett. 107, 041601 (2011).

[32] M. Tissier and G. Tarjus, Phys. Rev. B 85, 104203 (2012).

[33] G. Tarjus, I. Balog, and M. Tissier, Europhys. Lett. 103, 61001 (2013)

[34] J.-C. Anglès d'Auriac, M. Preissmann, and R. Rammal, J. Phys. Lett. 46, 173 (1985).

[35] A. V. Goldberg and R. E. Tarjan, J. Assoc. Comput. Mach. 35, 921 (1988).

[36] A. T. Ogielski, Phys. Rev. Lett. 57, 1251 (1986).

[37] J.-C. Anglès d'Auriac and N. Sourlas, Europhys. Lett. 39, 473 (1997).

[38] M. R. Swift, A. J. Bray, A. Maritan, M. Cieplak, and J. R. Banavar, Europhys. Lett. 38, 273 (1997).

[39] N. Sourlas, Comput. Phys. Commun. 121-122, 183 (1999); Proceedings of the Europhysics Conference on Computational Physics (CCP, Granada, 1998).

[40] A. K. Hartmann and U. Nowak, Eur. Phys. J. B 7, 105 (1999).

[41] A. A. Middleton, Phys. Rev. Lett. 88, 017202 (2001).

[42] A. K. Hartmann and A. P. Young, Phys. Rev. B 64, 214419 (2001).

[43] A. A. Middleton and D. S. Fisher, Phys. Rev. B 65, 134411 (2002).

[44] I. Dukovski and J. Machta, Phys. Rev. B 67, 014413 (2003).

[45] Y. Wu and J. Machta, Phys. Rev. Lett. 95, 137208 (2005).

[46] Y. Wu and J. Machta, Phys. Rev. B 74, 064418 (2006).

[47] N. G. Fytas and V. Martín-Mayor, Phys. Rev. Lett. 110, 227201 (2013).

[48] M. Picco and N. Sourlas, J. Stat. Mech. (2014) P03019.

[49] A. K. Hartmann, Phys. Rev. B 65, 174427 (2002).

[50] A. A. Middleton, arXiv:cond-mat/0208182.

[51] B. Ahrens and A. K. Hartmann, Phys. Rev. B 83, 014205 (2011).

[52] M. Picco and N. Sourlas, Europhys. Lett. 109, 37001 (2015).

[53] On the other hand, $0 \leq 2 \eta-\bar{\eta}$ is valid for all $D$ [30].

[54] See Supplemental Material at http://link.aps.org/ supplemental/10.1103/PhysRevLett.116.227201 for the parameters of the simulations; evidence for subleading corrections to scaling; $\chi^{2}$ for combined fits; details on the extrapolation to $L=\infty$ for $\xi^{\text {(dis) }} / L, U_{4}$ and $\nu$.

[55] D. J. Amit and V. Martín-Mayor, Field Theory, the Renormalization Group and Critical Phenomena, 3rd ed. (World Scientific, Singapore, 2005).

[56] M. Nightingale, Physica (Amsterdam) 83A, 561 (1976).

[57] H. G. Ballesteros, L. A. Fernandez, V. Martín-Mayor, and A. Muñoz Sudupe, Phys. Lett. B 378, 207 (1996).

[58] H. G. Ballesteros, L. A. Fernández, V. Martín-Mayor, A. Muñoz Sudupe, G. Parisi, and J. J. Ruiz-Lorenzo, Phys. Rev. B58, 2740 (1998). 